\title{
Opioids and Ethics: Is Opioid-Free the Only Responsible Arthroplasty?
}

\author{
Travis N. Rieder, PhD
}

Received: 14 September 2018/Accepted: 8 November 2018/Published online: 13 December 2018

(C) Hospital for Special Surgery 2018

\section{Opioids and Ethics?}

Against the backdrop of the US opioid epidemic, clinicians, hospitals, lawmakers, and the public are very concerned about the role of opioids in pain management. Although the majority of today's opioid overdose deaths involve heroin, illicit fentanyl, and polypharmacy [2], it is undeniable that reckless overprescribing helped fuel today's crisis [5]. As a result, a common question is: how can we reduce opioid prescribing? Or in the language of Toward Opioid-Free Arthroplasty: A Leadership Forum, the June 2018 gathering that inspired this special issue of HSS Journal, which I attended (Fig. 1): how do we move toward "opioid-free" medicine-in this case, opioid-free arthroplasty?

Here, I would like to make two points about the ethics of opioid prescribing during the US overdose epidemic. First is the conceptual point that "opioid-free" arthroplasty is not obviously the appropriate goal, nor is "reduced prescribing"; rather, we should aim for responsible prescribing, and whether that entails opioid-free practice is a contingent matter. Second, I would like to give more concrete guidance on responsible opioid prescribing in arthroplasty. Simply because a need for opioids exists, we should not resign ourselves to their predictable risks and harms. There is significant room for improvement.

Electronic supplementary material The online version of this article (https://doi.org/10.1007/s11420-018-9651-3) contains supplementary material, which is available to authorized users.

T. N. Rieder, $\operatorname{PhD}(\bowtie)$

Berman Institute of Bioethics, Johns Hopkins University,

1809 Ashland Ave,

Baltimore, MD 21205, USA

e-mail: Trieder@jhu.edu

\section{From "Opioid-Free" to "Opioid-Responsible" Arthroplasty}

Concerns with opioid overprescribing arose because of the harms it can and has caused. For two decades, overprescribing has contributed to increased rates of opioid use disorder and a skyrocketing overdose death rate [5]. Our concern with overprescribing, then, is a concern with the ethics of prescribing: providing more of a dangerous drug than needed risks harm to the patient and to public health and so is irresponsible.

As we recognize this point, it may become obvious that no number of pills or of morphine equivalents - and that includes the number zero- should be the aim as we seek to change practice. It is not the case that the risk of overprescribing means we should aim to eliminate opioids. What I propose, rather, is prescribing an appropriate amount of opioids, whatever that turns out to be. It is possible that appropriate opioid prescribing would involve opioid-free analgesia, but it is not obviously so.

So is opioid-free the only responsible arthroplasty?

It is easy to see why a significant decrease in opioid prescribing may be desired. Around 6\% of opioid-naïve patients who receive opioids after surgery go on to develop persistent opioid use [1,8]. Persistent use raises worries about addiction and the risk of overdose, but persistent opioid use is also, itself, a negative outcome, given the adverse effects and the risk/benefit profile of opioids. Thus, if surgeons were able to perform total joint arthroplasty (TJA) without opioid exposure, a significant post-surgical complication would be prevented. This is a good reason to explore the possibility.

On the other hand, discussion of opioid therapy against the backdrop of the US opioid crisis too often hinges on the risks of opioids as their only relevant feature. But it is also true that opioids are powerful analgesics, especially for certain forms of severe pain. TJA can be incredibly painful, and the acute post-surgical pain is-like the harms associated with opioid use - a genuine moral consideration. 


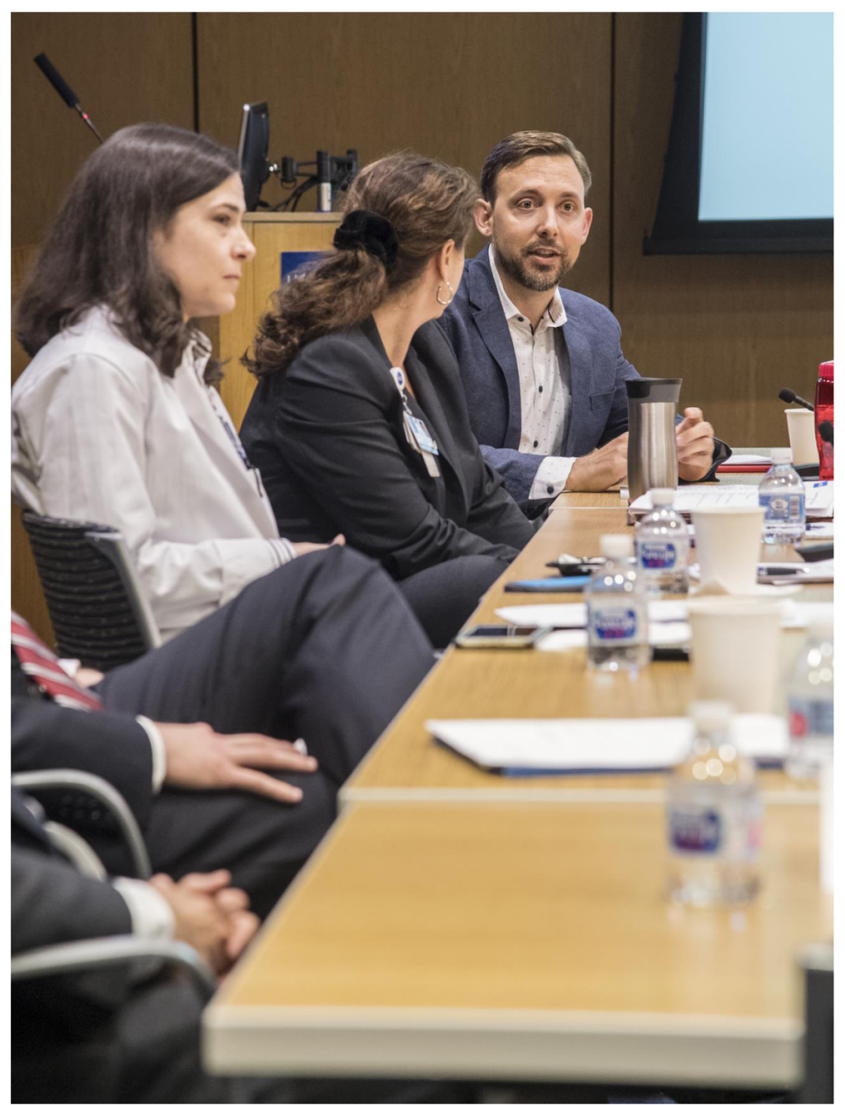

Fig. 1. Travis Rieder, $\mathrm{PhD}$, talks with other experts at the invitational symposium, Toward Opioid-Free Arthroplasty: A Leadership Forum, held at HSS in June 2018. "If no one knows whose job it is to protect patients from the risks of opioids," writes Rieder, "those risks are much more likely to materialize." Photograph by Robert Essel.

Allowing patients to suffer when they do not need to is a cost, and while it does not mean that all pain should be treated with powerful drug therapies, it does mean it must be a consideration when assessing the risk/benefit calculus for opioid therapy.

Pain management is not as simple a task as surgeons might like. Their blades cause considerable suffering, and opioids both provide a means to mitigate that suffering and carry substantial risk. Managing post-surgical pain requires weighing competing values, and as is often the case the clinician cannot weigh those values alone; doing so would be unjustifiably paternalistic. Patients have different beliefs, concerns, and fears about pain, medicine, and the risks of dependence and addiction, and their subjective considerations matter. As a result, pain management decisions are not only complex (due to competing values) but also dependent on the patient's values.

For these reasons, I am skeptical that responsible analgesia for TJA will always be opioid-free. Opioids can address the severe pain of these procedures well, especially in opioid-naïve patients. Yes, there are risks to opioid use, but there are risks to most of what is done in medicine. If the pain is severe enough, it does not seem a priori unreasonable for a patient to value some degree of pain relief over mitigating those risks. Of course, some patients (for instance, those in recovery from opioid use disorder) may be willing to forgo opioid analgesia, and that also seems reasonable. This argues for the idea that responsible analgesia can be opioid-free - not that it always should be.

\section{Improving Care while Continuing to Use Opioids}

If I am correct in my suspicion that we are not yet ready for opioid-free as the only responsible analgesia for arthroplasty, then surgeons are justified in continuing to prescribe opioids for these procedures. However, it is crucial to resist a move that is made far too often-from the claim that using opioids is permissible to a lack of concern with how they are used. Remember that the goal is ethically appropriate analgesia, and there is no reason to think that medical ethics would be silent on the details of opioid use, just because it is permissible to use them. Therefore, I would like to make some concrete recommendations on opioid use for TJA, moving from the most obvious to the more subtle and complex.

The most obvious recommendation for improving opioid-related complications after surgery is to use no more pain medication than necessary for mitigating the very worst of a patient's suffering. Although this seems commonsensi$\mathrm{cal}$, it is crucial to recognize both how important this advice is and how much better medicine could do in living up to it.

Consider first the importance of using as little pain medication as possible. More distressing than the $6 \%$ risk of new, persistent use after an initial exposure to opioids in opioid-naïve post-surgical patients is that an initial prescription of at least 10 days increases the risk to more than $15 \%$; after 31 days, it soars to $30 \%$ [8]. That means that nearly one-third of those given more than a 1-month prescription will be taking opioids 1 year later [8]. This sort of jump in risk justifies being very concerned not to prescribe more than is necessary.

Fortunately, evidence is beginning to accumulate demonstrating that even minor interventions can drastically reduce post-surgical prescribing. For instance, a team at the University of Michigan noted that the average prescription after gall bladder surgery was 250 morphine milligram equivalents (MME) and asked patients how many pills they actually used [3]. When patients said they used on average less than one eighth of that prescription (30 MME), physicians were educated and told patients that they would likely need very few pills. As a result, the average prescription dropped from 250 to $75 \mathrm{MME}$, with no increase in refill requests. In short, just educating prescribers on how few pills are actually necessary, and then preparing patients to take fewer pills, resulted in a drastic reduction in the number of pills going out the door. Based on similar data, the Michigan team has published evidence-based prescribing guidelines for several surgeries, and they are adding more all the time (see the website of the Opioid Prescribing Engagement Network: https://opioidprescribing.info).

There is good reason to think, then, that prescribers can do better at dispensing appropriate amounts of opioids, thereby avoiding some new cases of persistent opioid use. However, equally important is the need for opioid 
management. Opioids are not simple medications, and managing them does not end with prescribing them.

Consider the following two cases, one an orthopedic trauma case and the other an arthroplasty case. Both provide lessons relevant to post-surgical prescribing.

\section{Limb Salvage}

My journey into the ethics of opioid prescribing began with consideration of a trauma case resulting in opioid-related complications. Patient T was involved in a motorcycle accident and found himself in a limb-salvage situation. He underwent five major surgeries, received no warnings or counseling concerning his pain management regimen, and developed a profound dependence on opioids in 2 months. When the time came to stop the medication, he was given the bad advice to taper off approximately $170 \mathrm{MME}$ in just 4 weeks, dropping a quarter of the daily dose each week. Following these instructions, he went into terrible, acute withdrawal, suffering greatly and eventually becoming deeply depressed and suicidal. Despite his significant privilege and supportive family, patient $\mathrm{T}$ was not able to find a physician able or willing to manage his taper in a way that would minimize his suffering.

Dozens of clinicians in three hospitals were willing to write opioid prescriptions for patient $\mathrm{T}$, but none could manage those prescriptions over the long term [7].

\section{Bilateral Knee Arthroplasty}

A second patient-patient $\mathrm{J}$ - happened to know patient $\mathrm{T}$ and so was rather concerned when she learned she needed both knees replaced. She prepared herself for a painful recovery and committed to taking as little pain medication as necessary. Her surgeon prescribed $1207.5-\mathrm{mg}$ pills of hydrocodone/acetaminophen (Norco). Due to her diligence, she reduced the number of pills she took to just two a day during the second week of recovery, tapering to zero in the third week. In the days following her last pill, she experienced minor withdrawal symptoms, which she would not have identified had she not know patient T. Since she was able to identify them and knew they would pass, she managed not to resume opioid therapy. At the end of her recovery, she had used 47 pills out of her bottle of 120 .

\section{Analysis}

I know a lot about these two cases, as they are quite personal ones- I am patient T, and my mom is patient J. And these cases come nowhere near to exhausting my first-hand awareness of how little physicians know about preparing patients for opioid use, creating an exit strategy, and managing a course of opioid therapy. Yet without these crucial prescribing skills, clinicians provide access to a medication that carries the risks of tolerance, dependence, and addiction, without taking responsibility for minimizing those risks. These are not minor complications, and prescribers must learn how to provide access to these medications in a way that lessens their hazards.

These cases can elucidate the data on new, persistent opioid use after surgery. Dependence can develop quickly, and when it does, stopping the medication hurts. Patients who are not educated or monitored by competent clinicians may not realize they are experiencing withdrawal; one symptom of withdrawal is hyperalgesia, and so tapering or stopping opioids can convince patients that they are not ready to stop. By prescribing more pills than necessary surgeons can further the notion that the patient need not stop taking the medication. After all, if you have 73 pills remaining in a bottle of 120 , and going without hurts, you might believe that there is no reason to stop. Surely, the doctor would not have prescribed those pills if they were not needed or were dangerous, right?

These two cases also highlight a subtler set of prescribing obligations. In addition to prescribing an appropriate dosage, surgeons must counsel patients on pain and recovery, helping them to prepare to hurt. Eliminating pain completely would require significant opioid doses, but most people can put up with some pain for a limited time. This counseling must also include an agreed-upon exit strategy. Patients need to know when and how to stop taking their medications, and the longer they need them (for more complicated surgeries or for surgeries involving multiple procedures), the more likely it is that dependence will pose a challenge to recovery.

Not all of the work can be done prior to surgery; patients require monitoring after surgery, to ensure that they adhere to the plan and receive necessary support. This monitoring cannot wait for a visit a month or two after surgery; most patients should be off the medication long before that. Prescribing clinicians cannot fix a problem they never see, which means that someone must contact the patient regularly to discuss the pain management protocol.

If such interventions can head off potential problems, they also call for integrated services. Recognizing that a patient at post-operative weeks 2 and 3 is taking too much pain medication requires action, but perhaps not one that every surgeon or nurse is trained to deal with. Such patients may need a behavioral health assessment by a clinician trained to determine the appropriateness of the current course of treatment. Is the continued demand for opioids the result of pain from a complication, or is this a budding risk of developing opioid use disorder requiring specialized therapy? A surgical department, then, needs both the capacity to make behavioral health assessments and the relationships to pain management and addiction services to provide consultation and hand-off, as needed [4, 6, 9].

In short, the understandable focus on prescribing the correct number of pills risks overlooking the complexity of thoroughly responsible prescribing. Responsible opioid prescribing involves at least the following: (1) pre-surgical counseling and education of a patient who will receive opioid therapy; (2) prescribing an evidence-based amount that minimizes the risk of new, persistent opioid use; and (3) close management of the patient for as long as therapy continues, ensuring that the patient adheres to the plan and looking for signs that the patient needs a hand-off. These are significant obligations, but also sensible, considering the dangers. 


\section{Who Is Responsible for Ethical Prescribing and Management of Opioids?}

If my argument so far is on track, then I have established that patients have a right to fairly extensive services when they are prescribed opioids for post-surgical pain, which means that someone has the obligation to provide those services. But who, precisely, has these obligations?

A plausible answer is that the prescriber of the opioids is responsible because he or she is the one who provides access to the dangerous medication. And so the relevant principle would be something like: If you prescribe a medication that has foreseeable harmful side-effects, you are obligated to mitigate those harms [7]. This principle works well in many cases of prescribing, such as with family physicians. There is a complication in surgery, however, in that a whole team of clinicians works with the patient. While the surgeon performs the procedure, nurse practitioners (NPs) or physician assistants (PAs) may write discharge prescriptions and refills. So if the responsibility falls to whoever signs the prescription, it would be an NP or PA rather than the treating physician.

Does this seem like the right delineation of responsibility? Asking the question brings into tension two plausible ideas about responsible prescribing: first, that whoever actually signs a prescription bears some responsibility for managing that prescription; and second, that in the case of surgery, the operating physician is responsible for "the treatment" of a patient in some holistic sense, and that this includes mitigating the risks of harmful outcomes.

Pointing out the different ways of distributing responsibility for opioid therapy raises the question of opioid prescribing ethics to a structural level. We need not ask only who is responsible for managing the risks of opioids but also who should be responsible. How, that is, should a hospital, surgical center, or department be organized so that this set of obligations gets discharged reliably and efficiently?

There is no obviously correct answer to this question. An institution could decide to hold surgeons responsible for managing opioids, since they are the clinicians guiding overall treatment. Or it could decide that this is an inefficient use of resources, given the cost of a surgeon's time, and instead assign it to a team of NPs or PAs. There are no doubt other reasonable options. However such an institution is organized, though, the relevant parties would need to be educated to live up to these obligations and to be free to organize patient encounters in a way that aids their effort (making pain management check-in calls every few days, for instance).

The ethics of opioid prescribing, in other words, does not determine an appropriate model for discharging opioidrelated obligations. It does, however, make clear that a model must be chosen and acted on. It is likely that at many institutions, no one claims responsibility for these obligations, and there is no enforceable institutional understanding of the distribution of the responsibility.

And that is the outcome that is unjustifiable. If no one knows whose job it is to protect patients from the risks of opioids, those risks are much more likely to materialize. That is the precise lesson of my case studies.
Although our forum was titled "Toward Opioid-Free Arthroplasty," I have argued against opioid-free as the only responsible arthroplasty. Given the risks of opioids, it would be good if surgeons eventually can perform surgery without them; at present, though, we must recognize that opioid therapy can have significant benefits for some arthroplasty patients.

Recognizing this does not, however, mean that we should give up on improving opioid prescribing. Responsible opioid use requires solid evidence on prescribing habits, which would almost certainly reduce total prescribing [3]. Further, the writing of a prescription is not the only ethically relevant component of prescribing. Responsible opioid prescribing also requires medication management, including educating and counseling the patient and intervening as necessary when therapy does not go as planned.

Opioid-free arthroplasty may not, then, be the only responsible arthroplasty. Indeed, responsible opioid use in TJA requires a re-envisioning of the ethical division of labor in surgery. Surgery requires a team approach including members charged with overseeing opioid prescriptions. All team members must be informed of this role, and there must be an appropriate system of accountability.

Acknowledgments My thanks to all of the attendees of Toward Opioid-Free Arthroplasty: A Leadership Forum, held at HSS in June 2018, for constructive comments and feedback. Special thanks go to Seth Waldman, MD, for two discussions on the role of bioethics in orthopedic surgery and to Joy Jacobson for feedback on multiple drafts of this article.

\section{Compliance with Ethical Standards}

Conflict of Interest: Travis N. Rieder, $\mathrm{PhD}$, declares that he has no conflicts of interest.

Human/Animal Rights: N/A

Informed Consent: N/A

Required Author Forms Disclosure forms provided by the author are available with the online version of this article.

\section{References}

1. Brummett CM, et al. New persistent opioid use after minor and major surgical procedures in US adults. JAMA Surg. 2017;152(6):e170504.

2. Seth P, Scholl L, Rudd RA, Bacon S. Overdose deaths involving opioids, cocaine, and psychostimulants-United States, 20152016. MMWR Morb Mortal Wkly Rep. 2018;67:349-358.

3. Howard R, Waljee J, Brummett C, Englesbe M, Lee J. Reduction in opioid prescribing through evidence-based prescribing guidelines. JAMA Surg. 2018;153(3):285-287.

4. Katz J, et al. The Toronto General Hospital Transitional Pain Service: development and implementation of a multidisciplinary program to prevent chronic postsurgical pain. J Pain Res. 2015;8:695-702. 
5. Kolodny A, et al. The prescription opioid and heroin crisis: a public health approach to an epidemic of addiction. Annu Rev Public Health. 2015;36:559-574.

6. Philips BD, et al. Creation of a novel recuperative pain medicine service to optimize postoperative analgesia and enhance patient satisfaction. HSS J. 2010;6(1):61-65.

7. Rieder TN. In opioid withdrawal, with no help in sight. Health Aff (Millwood). 2017;36(1):182-185.
8. Shah A, Hayes CJ, Martin BC. Characteristics of initial prescription episodes and likelihood of long-term opioid use-United States, 2006-2015. MMWR Morb Mortal Wkly Rep. 2017;66(10):265-269.

9. Soffin EM, Waldman SA, Stack RJ, Liguori GA. An evidence-based approach to the prescription opioid epidemic in orthopedic surgery. Anesth Analg. 2017;125(5):17041713. 